\title{
The majority coloring of the join and Cartesian product of some digraph
}

\author{
Mei Shi, Weihao Xia, Mingyue Xiao, and Jihui Wang* \\ School of Mathematical Sciences, University of Jinan, Jinan, China, 250022
}

\begin{abstract}
A majority coloring of a digraph is a vertex coloring such that for every vertex, the number of vertices with the same color in the outneighborhood does not exceed half of its out-degree. Kreutzer, Oum, Seymour and van der Zyper proved that every digraph is majority 4colorable and conjecture that every digraph has a majority 3-coloring. This paper mainly studies the majority coloring of the joint and Cartesian product of some special digraphs and proved the conjecture is true for the join graph and the Cartesian product. According to the influence of the number of vertices in digraph, we prove the majority coloring of the joint and Cartesian product of some digraph.
\end{abstract}

\section{Introduction}

For a digraph $D=(V(D), A(D))$ and every vertex $v \in V(D)$, let $N^{+}(v)\left(N^{-}(v)\right)$ denote the out-neighborhood(in-neighborhood) of $v$ in $D \cdot d^{+}(v)\left(d^{-}(v)\right)$ is the size of $N^{+}(v)\left(N^{-}(v)\right)$. A directed arc with tail $u$ and head $v$ is denoted by $u v$, vertex $u$ and $v$ are both called end of $(u, v)$. For a subset $V_{1} \subseteq V$,we let $D\left[V_{1}\right]$ denote the induce subdigraph by the vertices of $V_{1}$.

A proper vertex coloring of a digraph $D$ is an assignment $c: V(D) \rightarrow\{1,2, \cdots, k\}$ such that there exists no monochromatic arc, then we say that $D$ is proper $k$-coloring. The least number $k$ that satisfies proper vertex coloring is denoted by $\chi(D)$.

A majority coloring of a digraph $D$ is an assignment $c: V(D) \rightarrow\{1,2, \cdots, k\}$ such that for every vertex $v \in V(D)$, at most half the out-neighbors of $v$ receive the same color as $v$, then we say that $D$ is majority $k$-colorable. The least number $k$ that satisfies this color is denoted by $\chi_{m}(D)$.The number of vertices that receive the color $i$ in the outneirhborhood of $v$ in $D$ is denoted by $n_{D}^{+}(c, v, i)$. The majority coloring of digraph was

\footnotetext{
* Corresponding author: wangjihui@unjn.edu.cn
} 
first introduced and studied by S. Kreutzer, Oum, P. Seymour, D. van der Zypen and D.R. Wood[1], who showed that every digraph is majority 4-colorable. They proved this result on the basis that every acyclic digraph is majority 2-colorable. A majority coloring of odd directed cycle must be a proper vertex coloring, three colors are necessary. Therefore, they proposed the following conjecture:

Conjecture 1.1([1]). Every digraph is majority 3-colorable.

Although this conjecture has not been fully resolved, S. Kreutzer et al.[1] studied some special digraphs. The conjecture is true for the digraphs with certain restrictions on outdegree or in-degree. M. Anastos, A. Lamaison, R. Steiner and T. Szabo[2] showed the following theorem:

Theorem 1.2([2]). Let $D$ be a digraph such that $\chi(D) \leq 6$. Then $D$ is majority 3 coloring.

We call a digraph $k$-majority choosable, if for any assignment of lists of sizes at least $k$ to the vertices, we can choose colors from the respective lists such that the arising coloring is a majority coloring. Anholcer, Bosek and Grytczuk[3] gave a beautiful proof to show that every digraph is majority 4-choosable(not only majority 4-colorable). M. Anastos, A. Lamaison, R. Steiner and T. Szabo[2] showed the following theorem:

Theorem 1.3[2] If $\Delta^{+}(D) \leq 4$ or $\Delta(U(D)) \leq 6$ or $\Delta(D) \leq 7$, then $D$ is majority 3-choosable.

Theorem 1.4[2] Let $D$ be a digraph whose underlying undirected graph is 6-choosable. Then $D$ is majority 3-choosable. In particular any digraph with a 5-degenerate underlying graph is majority 3 -choosable.

Theorem 1.5[2] If $D$ is a digraph without odd directed cycle, then $D$ is majority 2choosable.

A. Girão et al. [4] and F. Knox and R. Sámal[5] investigated a natural generalization of majority colorings: For any $\alpha \in[0,1]$, define an $\alpha$-majority coloring of a digraph $D$ to be a vertex-coloring in which for every vertex $v$, at most $\alpha \cdot d^{+}(v)$ vertices in $N^{+}(v)$ have the same color as $v$. If such a coloring can be found for any assignment of lists of sizes at least $k$ to the vertices, call the digraph $\alpha$-majority $k$-choosable. A. Girão et al. [4] and F. Knox and R. Sámal[5] proved both that for every integer $k \geq 1$, every digraph is $\frac{1}{k}$-majority $2 k$-choosable. A. Girão et al. [4] proposed the following conjecture:

Conjecture 1.6[4] For every integer $k \geq 1$, every digraph is $\frac{1}{k}$-majority $(2 k-1)$ choosable.

It is obvious that even directed cycles and directed paths are majority 2-colorable, and odd directed cycles are majority 3-colorable. In this paper, We study the majority coloring of the join and Cartesian product of some digraphs. In Section 2, we prove several results about the majority coloring of the join of some digraphs. In Section 3, we prove the result about the majority coloring of the Cartesian product of some digraphs. 


\section{The majority coloring of the join of some digraphs}

The join of $G$ and $H$ is a graph where the vertex set is $V=V(G) \cup V(H)$ and the edge set is $E=\{u v \mid u \in V(G), v \in V(H)\} \cup E(G) \cup E(H)$. We denote the join of $G$ and $H$ by $G \mathrm{~V} H$.

Let $D_{1}, D_{2}$ be digraphs, we can denote the join of $D_{1}, D_{2}$ by $D_{1} \overrightarrow{\mathrm{V}} D_{2}$, this mean that the arc set of $D_{1} \overrightarrow{\mathrm{V}} D_{2}$ is $A=\left\{u v \mid u \in V\left(D_{1}\right), v \in V\left(D_{2}\right)\right\} \cup A\left(D_{1}\right) \cup A\left(D_{2}\right)$, the vertex set is still $V=V\left(D_{1}\right) \cup V\left(D_{2}\right)$. Obviously, the out-degree of vertex in $D_{2}$ remains unchanged. Therefore, for majority coloring of the join $D_{1} \overrightarrow{\mathrm{V}} D_{2}$, we just have to consider the change of the out-degree of the vertex in $D_{1}$.

Let $m, n \in \mathrm{N}$, and $m \geq 2, n \geq 3, P_{m}=u_{1} u_{2} \cdots u_{m}$ is a directed path with $m$ vertices, $C_{n}=v_{1} v_{2} \cdots v_{n}$ is a directed cycle with $n$ vertices. Next, we study the majority coloring of the join of directed paths and directed cycles.

Theorem 2.1 Let $m, n \in \mathrm{N}$, and $m, n \geq 2, P_{m} \overrightarrow{\mathrm{V}} P_{n}$ is majority 2-colorable.

Proof. We know $\chi\left(P_{l}\right)=2, \chi_{m}\left(P_{l}\right)=2$, for any directed path $P_{l}, l \in \mathrm{N}$. Let $P_{m}=u_{1} u_{2} \cdots u_{m}, P_{n}=v_{1} v_{2} \cdots v_{n}$. For $v \in V\left(P_{n}\right)$, the out-neighborhood of $v$ remains unchanged, hence a proper 2-coloring of $P_{n}$ is a majority coloring $c_{P_{n}}$ of $P_{n}$. Next, let's consider vertices in directed path $P_{m}$. For every $u_{i} \in V\left(P_{m}\right)$,

$$
\underset{P_{m} \mathrm{~V} P_{n}}{N^{+}}\left(u_{i}\right)=\left\{u_{i+1}\right\} \bigcup V\left(P_{n}\right),(i=1,2, \cdots, m-1) ; \underset{P_{m} \mathrm{~V} P_{n}}{N^{+}}\left(u_{m}\right)=V\left(P_{n}\right) .
$$

If $n$ is even, $n_{P_{m} \mathrm{~V} P_{n}}\left(c_{P_{n}}, u_{m}, i\right)=\frac{n}{2}(i=1,2)$, we only need to perform proper vertex coloring on $P_{m}$.

If $n$ is odd, the number of vertices of the two colors in $P_{n}$ are $\frac{n-1}{2}$, $\frac{n+1}{2}$, respectively. For vertex $u_{m} \in V\left(P_{m}\right)$, we suppose $n_{P_{m} \mathrm{~V} P_{n}}\left(c_{P_{n}}, u_{m}, 1\right)=\frac{n-1}{2}$, $n_{P_{m} \mathrm{~V} P_{n}}^{+}\left(c_{P_{n}}, u_{m}, 2\right)=\frac{n+1}{2}$. We know that $\underset{P_{m} \overrightarrow{\mathrm{V} P} P_{n}}{N^{+}}\left(u_{m}\right)=V\left(P_{n}\right)$. Therefore we color $u_{m}$ with color 1 . According to this, we alternate the coloring of $u_{i}(i=1, \cdots, m-1)$ with color $\{1,2\}$, then we can obtain a majority 2-coloring of $P_{m} \overrightarrow{\mathrm{V}} P_{n}$. This proves the theorem. 
Theorem 2.2 Let $m, n \in \mathrm{N}$, and $m \geq 2, n \geq 3$, if $n$ is even, then $P_{m} \overrightarrow{\mathrm{V}} C_{n}$ is majority 2-colorable; if $n$ is odd, then $P_{m} \overrightarrow{\mathrm{V}} C_{n}$ is majority 3-colorable.

Proof. Let $P_{m}=u_{1} u_{2} \cdots u_{m}, C_{n}=v_{1} v_{2} \cdots v_{n}$. Frist, we consider the case if $n$ is even, $C_{n}$ is an even directed cycle, so it is majority 2-colorable, and satisfied $n_{P_{m} \overrightarrow{\mathrm{V}} C_{n}}^{+}\left(c_{C_{n}}, u_{m}, i\right)=\frac{n}{2}(i=1,2)$. We have that $n \leq d^{+}\left(u_{i}\right) \leq n+1$, for every $u_{i} \in V\left(P_{m}\right)(i=1, \ldots, m)$. Let $c_{P_{m}}\left(u_{i}\right)=1(i$ is odd $), c_{P_{m}}\left(u_{i}\right)=2(i$ is even $)$. It is the majority coloring of the join $P_{m} \overrightarrow{\mathrm{V}} C_{n}$ that we want.

If $n$ is odd, $C_{n}$ is an odd cycle, and the out-neighborhood of $v_{j}(j=1, \cdots, n)$ remains unchanged in $C_{n}$, so $C_{n}$ must be majority 3-colorable,and the majority coloring is also a proper vertex coloring. We perform proper vertex coloring on $P_{m}$ with color $\{1,2\}$. Thus, we can obtain that

$$
\left\lfloor\frac{n}{3}\right\rfloor \leq q \leq\left\lfloor\frac{n}{3}\right\rfloor+1, q \leq \frac{d^{+}\left(u_{i}\right)}{2}(i=1, \cdots, m),
$$

for every vertex $u_{i}(i=1, \cdots, m)$, where $q$ denotes the number of $u_{i}$ with the same color in the out-neighborhood of $u_{i}$ in $P_{m} \overrightarrow{\mathrm{V}} C_{n}$. Thus, $P_{m} \overrightarrow{\mathrm{V}} C_{n}$ is majority 3-colorable. This concludes the proof of the theorem.

Theorem 2.3 Let $m, n \in \mathrm{N}$, and $m \geq 2, n \geq 3$, if $m$ is odd and $n$ is even, then $C_{m} \overrightarrow{\mathrm{V}} P_{n}$ is majority 3-colorable; otherwise $C_{m} \overrightarrow{\mathrm{V}} P_{n}$ is majority 2-colorable.

Proof. Let $C_{m}=u_{1} u_{2} \cdots u_{m}, P_{n}=v_{1} v_{2} \cdots v_{n}$. We know that $P_{n}$ is majority 2colorable. If $n$ is even, $n_{C_{m} \mathrm{~V} P_{n}}\left(c_{P_{n}}, u_{m}, i\right)=\frac{n}{2}(i=1,2)$. We have that $d_{C_{m} \overrightarrow{\mathrm{V}} P_{n}}^{+}(u)=n+1$, for every vertex $u \in V\left(C_{m}\right)$. Thus, we only need to ensure that the vertex coloring of $C_{m}$ is majority colorable. If $m$ is odd, then $C_{m}$ is majority 3-colorable, thus $C_{m} \overrightarrow{\mathrm{V}} P_{n}$ is majority 3-colorable. If $m$ is even, then $C_{m}$ is majority 2-colorable, thus $C_{m} \overrightarrow{\mathrm{V}} P_{n}$ is majority 2-colorable.

If $n$ is odd, we have that $\left|n_{C_{m} \overrightarrow{\mathrm{V}} P_{n}}^{n_{P_{n}}},\left(c_{P_{m}}, 1\right)-n_{C_{m} \overrightarrow{\mathrm{V}} P_{n}}^{+}\left(c_{P_{n}}, u_{m}, 2\right)\right|=1$. We may suppose that $n_{C_{m} \mathrm{~V} P_{n}}^{+}\left(c_{P_{n}}, u_{m}, 1\right)=\frac{n-1}{2}, n_{C_{m} \overrightarrow{\mathrm{V}} P_{n}}\left(c_{P_{n}}, u_{m}, 2\right)=\frac{n+1}{2}$. If $m$ is even, 
the proper 2-coloring of $C_{m}$ must be a majority 2-coloring. Then we can obtain the majority 2-coloring of $C_{m} \overrightarrow{\mathrm{V}} P_{n}$. If $m$ is odd, we color $u_{m}$ with color 1 . By the coloring of $u_{m}$,we alternate the coloring of $V\left(C_{m}\right)$ with color $\{1,2\}$, then there exists a monochromatic arc, we can suppose that $u_{m} u_{1}$ is a monochromatic arc. The number of $u_{m}$ with the same color in the out-neighborhood of $u_{m}$ in $C_{m} \overrightarrow{\mathrm{V}} P_{n}$ is $\left(\frac{n-1}{2}+1\right)=\frac{n+1}{2}$. Thus, $C_{m} \overrightarrow{\mathrm{V}} P_{n}$ is majority 2-colorable. This proves the theorem.

Theorem 2.4 Let $m, n \in \mathrm{N}$, and $m, n \geq 3$, if $m, n$ are even, then $C_{m} \overrightarrow{\mathrm{V}} C_{n}$ is majority 2-colorable; otherwise $C_{m} \overrightarrow{\mathrm{V}} C_{n}$ is majority 3-colorable.

Proof. Let $C_{m}=u_{1} u_{2} \cdots u_{m}, C_{n}=v_{1} v_{2} \cdots v_{n}$. First, we consider that $m, n$ are even, $C_{m}, C_{n}$ are even directed cycle, thus they are also majority 2-colorable. Thus a proper coloring of $C_{m} \overrightarrow{\mathrm{V}} C_{n}$ is a majority coloring.

Next, we consider that $n$ is even, $m$ is odd, $C_{n}$ is an even directed cycle, and the proper 2-coloring of $C_{n}$ must be a majority 2-coloring. We suppose that $C_{m} \overrightarrow{\mathrm{V}} C_{n}$ is majority 2-colorable. Then $V\left(C_{m}\right)$ has at most two colors. Because $m$ is odd, there must exist a monochromatic arc, we can suppose that $u_{m} u_{1}$ is a monochromatic arc. For vertex $u_{m}, d_{C_{m} \overrightarrow{\mathrm{V}} C_{n}}\left(u_{m}\right)=n+1$. The number of vertices with the same color of $u_{m}$ in the outneighborhood of $u_{m}$ in $C_{m} \overrightarrow{\mathrm{V}} C_{n}$ is $\left(\frac{n}{2}+1\right)>\frac{n+1}{2}$, a contradiction. Therefore $C_{m} \overrightarrow{\mathrm{V}} C_{n}$ is majority 3-colorable.

Finally, if $n$ is odd, $C_{n}$ is an odd directed cycle, thus the proper vertex coloring of $C_{m}$ must be majority 3-colorable. Regardless of the parity of $m, C_{m}$ is must majority 3colorable. Therefore $C_{m} \overrightarrow{\mathrm{V}} C_{n}$ is majority 3-colorable. This proves the claim.

\section{The majority coloring of the Cartesian product of some digraphs}

The Cartesian product of graph $G$ and $H$ is a graph that vertex set is

$$
V(G \times H)=\{(u, v) \mid u \in V(G), v \in V(H)\},
$$

the arc set is 


$$
E(G \times H)=\left\{(u, v)\left(u^{\prime}, v^{\prime}\right) \mid u=u^{\prime}, v v^{\prime} \in E(H) \text { or } v=v^{\prime}, u u^{\prime} \in E(G)\right\} .
$$

We denoted the Cartesian product of graph $G$ and $H$ by $G \times H$.

This definition is extended to digraphs: Let $D_{1}=\left(V_{1}, A_{1}\right), D_{2}=\left(V_{2}, A_{2}\right)$ be digraphs, the Cartesian product $D_{1} \times D_{2}$ is a digraph that the vertex set is

the arc set is

$$
V\left(D_{1} \times D_{2}\right)=\left\{(u, v) \mid u \in V_{1}, v \in V_{2}\right\},
$$

$$
A\left(D_{1} \times D_{2}\right)=\left\{(u, v)\left(u^{\prime}, v^{\prime}\right) \mid u=u^{\prime}, v v^{\prime} \in A_{2} \text { or } v=v^{\prime}, u u^{\prime} \in A_{1}\right\},
$$

where $(u, v)\left(u^{\prime}, v^{\prime}\right)$ is the arc from $(u, v)$ to $\left(u^{\prime}, v^{\prime}\right)$.

Let $m, n \in \mathrm{N}$, and $m \geq 2, n \geq 3, P_{m}$ is a directed path with $m$ vertices, $C_{n}$ is a directed cycle with $n$ vertices.

Next, we give the conclusion of the majority coloring of the Cartesian product composed of directed path and directed cycle.

Theorem 3.1 Let $m, n \in \mathrm{N}$, and $m, n \geq 2, P_{m} \times P_{n}$ is majority 2-colorable.

Proof. It is obviously that $d^{+}\left(u_{i}, v_{j}\right) \leq 2$, for each vertex $\left(u_{i}, v_{j}\right) \in V\left(P_{m} \times P_{n}\right)$. The proper 2-coloring of $P_{m} \times P_{n}$ must be a majority 2-coloring, hence $P_{m} \times P_{n}$ is majority 2-colorable. The theorem is proved.

We know that the Cartesian Product satisfies the commutative law, hence the majority coloring of $P_{m} \times C_{n}$ and $C_{n} \times P_{m}$ is the same. Therefore we only need to discuss one case of two cases, and suppose that we discuss $P_{m} \times C_{n}$. This prove the claim.

Theorem 3.2 Let $m, n \in \mathrm{N}$, and $m \geq 2, n \geq 3$, if $n$ is odd, $P_{m} \times C_{n}$ is majority 3colorable; otherwise $P_{m} \times C_{n}$ is majority 2-colorable.

Proof. Let $P_{m}=u_{1} u_{2} \cdots u_{m}, C_{n}=v_{1} v_{2} \cdots v_{n}$, then

$$
V\left(P_{m} \times C_{n}\right)=\left\{\left(u_{i}, v_{j}\right) \mid u_{i} \in V\left(P_{m}\right), v_{j} \in V\left(C_{n}\right), i=1, \cdots, m ; j=1, \cdots, n\right\} .
$$

For each vertex $\left(u_{i}, v_{j}\right) \in V\left(P_{m} \times C_{n}\right)(i=1, \cdots, m-1 ; j=1, \cdots, n)$, $d^{+}\left(\left(u_{i}, v_{j}\right)\right)=2$ and

$$
N^{+}\left(\left(u_{i}, v_{j}\right)\right)=\left\{\left(u_{i+1}, v_{j}\right),\left(u_{i}, v_{j+1}\right)\right\}(j \bmod \mathrm{n}) .
$$

For each vertex $\left(u_{m}, v_{j}\right)(j=1, \cdots, n) \quad, \quad d^{+}\left(\left(u_{m}, v_{j}\right)\right)=1 \quad$ and $N^{+}\left(\left(u_{m}, v_{j}\right)\right)=\left\{\left(u_{i+1}, v_{j}\right)\right\}(j \bmod \mathrm{n})$.

Let $V_{i}=\left\{\left(u_{i}, v_{j}\right) \mid j=1, \cdots, n\right\}, i=1, \cdots, m$, and $W_{j}=\left\{\left(u_{i}, v_{j}\right) \mid i=1, \cdots, m\right\}$, $j=1, \cdots, n$. Every $D\left[V_{i}\right]$ is a directed $n$ cycle. If $n$ is odd, then each $D\left[V_{i}\right]$ is an odd directed cycle, hence each $D\left[V_{i}\right]$ is majority 3 -colorable. We color every $D\left[V_{i}\right]$ in the following way: 


$$
\begin{gathered}
c\left(\left(u_{i}, v_{j}\right)\right)=0, j=0(\bmod 3) ; c\left(\left(u_{i}, v_{j}\right)\right)=1, j=1(\bmod 3) ; c\left(\left(u_{i}, v_{j}\right)\right)=2, \\
j=2(\bmod 3) .
\end{gathered}
$$

The above coloring is majority 3 -colorable of $P_{m} \times C_{n}$.

If $n$ is even, then $D\left[V_{m}\right]$ is an even directed cycle, and is majority 2-colorable. We color each $D\left[V_{i}\right]$ such that the coloring $c_{i}$ is peoper 2-colorable, and is majority 2colorable. Then $P_{m} \times C_{n}$ has a majority 2-coloring. The theorem is proved.

Theorem 3.3 Let $m, n \in \mathrm{N}$, and $m, n \geq 3, C_{m} \times C_{n}$ is majority 2-colorable.

Proof. According to the definition of Cartesian product, we know that $C_{m} \times C_{n}$ is a 2regular digraph i.e. $d^{+}\left(\left(u_{i}, v_{j}\right)\right)=d^{-}\left(\left(u_{i}, v_{j}\right)\right)=2$, for every vertex $\left(u_{i}, v_{j}\right) \in$ $V\left(C_{m} \times C_{n}\right) \quad$. Let $\quad V_{i}=\left\{\left(u_{i}, v_{j}\right) \mid j=1, \cdots, n\right\} \quad, \quad i=1, \cdots, m \quad$, and $W_{j}=\left\{\left(u_{i}, v_{j}\right) \mid i=1, \cdots, m\right\}, j=1, \cdots, n$.We color every vertex $\left(u_{i}, v_{j}\right)$ in the following way:

$c\left(u_{i}, v_{j}\right)=1, i, j$ are also odd or even;

$c\left(\left(u_{i}, v_{j}\right)\right)=0, i$ is odd and $j$ is even or $i$ is even and $j$ is odd.

If $m, n$ are also odd, $D\left(V_{i}\right), D\left(W_{j}\right)$ are odd directed cycles. We can know that the above coloring of $D\left[V_{m}\right]$ and $D\left[V_{1}\right]$ is the same, $D\left[W_{n}\right]$ and $D\left[W_{1}\right]$ is the same. For every vertex $\left(u_{i}, v_{j}\right) \in V\left(C_{m} \times C_{n}\right) \backslash\left(D\left[V_{m}\right] \cup D\left[W_{n}\right]\right)$, the number of vertices with the same color of $\left(u_{i}, v_{j}\right)$ in the out-neighborhood of $\left(u_{i}, v_{j}\right)$ in $C_{m} \times C_{n}$ is $q=0$.For every vertex $\left(u_{i}, v_{j}\right) \in\left(D\left[V_{m}\right] \cup D\left[W_{n}\right]\right) \backslash\left(u_{m}, v_{n}\right), q=1=\frac{1}{2} d^{+}\left(u_{i}, v_{j}\right)$, where $q$ denotes the number of vertices with the same color of $\left(u_{i}, v_{j}\right)$ in the out-neighborhood of $\left(u_{i}, v_{j}\right)$ in $C_{m} \times C_{n}$. For the vertex $\left(u_{m}, v_{n}\right)$, its out-neighbors $\left(u_{m}, v_{1}\right)$ and $\left(u_{1}, v_{n}\right)$ are the same color as it. We change the color of $\left(u_{m}, v_{1}\right)$. For the vertex $\left(u_{m}, v_{1}\right), q=1=\frac{1}{2} d^{+}\left(u_{m}, v_{1}\right)$, where $q$ represents the number of vertices with the same color of $\left(u_{m}, v_{1}\right)$ in the out-neighborhood of $\left(u_{m}, v_{1}\right)$ in $C_{m} \times C_{n}$. Therefore $C_{m} \times C_{n}$ is majority 2-colorable.

When at least one of $m$ and $n$ is even, first we consider that exactly one is even. We can suppose that $m$ is odd, $n$ is even. Every $D\left[V_{i}\right]$ is an even directed cycle, and every $D\left[W_{j}\right]$ is an odd directed cycle. The number of vertices with the same color of $\left(u_{i}, v_{j}\right)$ 
in the out-neighborhood of $\left(u_{i}, v_{j}\right)$ in $C_{m} \times C_{n}$ is $q=1$, for every vertex $\left(u_{i}, v_{j}\right) \in$ $V\left(C_{m} \times C_{n}\right)$. Thus $C_{m} \times C_{n}$ is majority 2-colorable. Finally, if $m$ and $n$ are also even, every $D\left[V_{i}\right]$ and $D\left[W_{j}\right]$ are also even directed cycle, the above coloring is proper 2colorable, and is also majority 2-colorable. Thus, $C_{m} \times C_{n}$ is majority 2-colorable. This proves the theorem.

This work was supposed by the National Natural Science Foundation of China (12071351) and the Natural Science Foundation of Shandong Provence (ZR2020MA043).

\section{References}

1. S. Kreutzer S. Oum, P. Seymour and D. van der Zypen 2016 Majority colorables of digraphs The electronic journal of combinatorics 24(2)

2. M. Anastos A. Lamaison R. Steiner and T. Szabó 2019 Majority colorings of sparse digraphs https://arxiv.org/abs/1911.01954

3. M. Anholcer B. Bosek and J. L. Grytczuk 2016 Majority choosability of digraphs Electron. J. Combin. 24(3)

4. A. Girâo T. Kittipassorn and K. Popielarz 2017 Generalised majority colourings of digraphs Graph Theory 26(6) P850-P855

5. F. Knox and R.Šámal 2017 Linear bound for majority colourings of digraphs https://arxiv.org/abs/1701.05715

6. D. van der Zyper Majority coloring for directed graphs 2016 https://mathoverflow.net/

7. V. Neumann-Lara 1982 The dichromatic number of a digraph Journal of combinatorial theory Series B 33 265-270

8. J. Bensmail A. Harutyunyan and N. K. Le 2018 List coloring digraphs Journal of Graph Theory 87(4) 492-508

9. A. Harutyunyan and B. Mohar 2012 Two results on the digraph chromatic number Discrete Mathematics 312(10) 1823-1826

10. N. Alon J. Bang-Jensen and S. Bessy 2019 Out-colourings of digraphs J. Graph Theory. DOI: $10.1002 /$ jgt.22476 